\title{
Cell Type-Dependent Trafficking of Neuropeptide Y- Containing Dense Core Granules in CNS Neurons
}

\author{
Prabhu Ramamoorthy, ${ }^{1}$ Qian Wang, ${ }^{1,2}$ and Matthew D. Whim ${ }^{1,2}$ \\ ${ }^{1}$ Department of Biology, Pennsylvania State University, State College, Pennsylvania 16802 and ${ }^{2}$ Department of Cell Biology and Anatomy, Louisiana State \\ University Health Sciences Center, New Orleans, Louisiana 70112
}

\begin{abstract}
Neuropeptide transmitters are synthesized throughout the CNS and play important modulatory roles. After synthesis in the neuronal cell body, it is generally assumed that peptides are transported to nonspecialized sites of release. However, apart from a few cases, this scenario has not been thoroughly examined. Using wild-type and NPY(GFP) transgenic mice, we have studied the subcellular distribution of neuropeptide Y (NPY), a prototypical and broadly expressed neuropeptide. NPY puncta were found in the dendrites and axons of hippocampal GABAergic interneurons in situ. In contrast in hypothalamic GABAergic interneurons, NPY was restricted to the axon. Surprisingly this differential trafficking was preserved when the neurons were maintained in vitro. When hippocampal and hypothalamic neurons were transfected with NPY-Venus, the distribution of the fluorescent puncta replicated the cell type-specific distribution of endogenous neuropeptide Y. The NPY puncta in the axons of hippocampal and hypothalamic neurons colocalized with the sites of classical transmitter release (identified by staining for synapsin and the vesicular GABAergic transporter, VGAT). In hippocampal neurons, most of the postsynaptic NPY puncta were clustered opposite synapsincontaining varicosities. When neurons were stained for a second neuropeptide, agouti-related protein, immunoreactivity was found in the axon and dendrites of hippocampal neurons but only in the axons of hypothalamic neurons, thus mimicking the polarized distribution of NPY. These results indicate that the trafficking of neuropeptide-containing dense core granules is markedly cell type specific and is not determined entirely by the characteristics of the particular peptide per se.
\end{abstract}

\section{Introduction}

Neuropeptide Y (NPY) is a widely distributed neuropeptide in the CNS and peripheral nervous system and modulates a variety of physiological processes including blood pressure, feeding, and anxiety (Blomqvist and Herzog, 1997). In contrast to classical transmitters, neuropeptides including NPY are synthesized in the cell body and stored within dense core granules. The sites of release of these transmitters are distinct. Classical transmitters are released at specialized presynaptic varicosities. In contrast, granule fusion can occur at the cell body, axon, and dendrites (Huang and Neher, 1996; Hartmann et al., 2001; Shakiryanova et al., 2006; Sobota et al., 2010).

Despite these observations, many aspects of neuropeptide trafficking remain unclear. In particular, there appears to be no clear pattern to their subcellular localization. Neuropeptides are found at presynaptic or postsynaptic sites, and some have been found at both. Thus, hypothalamic magnocellular neurons in the supraoptic and paraventricular nuclei synthesize oxytocin and vasopressin. In these cells, oxytocin-containing

Received June 10, 2011; revised July 29, 2011; accepted Aug. 19, 2011.

Author contributions: P.R., Q.W., and M.D.W. designed research; P.R., Q.W., and M.D.W. performed research; P.R., Q.W., and M.D.W. analyzed data; M.D.W. wrote the paper.

This work was supported by grants from the National Institutes of Health (R56DK080441 and R01DK080441) to M.D.W. We thank Dr. June Liu for critically reading this manuscript, Dr. Christophe Dubois for cerebellar cultures, and Dr. Charles Nichols and the Department of Pharmacology (LSU Health Sciences (enter) for the use of a confocal microscope.

Correspondence should be addressed to Matthew D. Whim, Department of Cell Biology and Anatomy, Louisiana State University Health Sciences Center (MEB 6142), 1901 Perdido Street, New Orleans, LA 70112. E-mail: mwhim@|suhsc.edu.

P. Ramamoorthy's present address: Department of Pharmacology and Toxicology, University of Kansas, Lawrence, KS 66045.

DOI:10.1523/JNEUROSCI.2933-11.2011

Copyright $\odot 2011$ the authors $\quad 0270-6474 / 11 / 3114783-06 \$ 15.00 / 0$ granules are found in the axon and dendrites. Axonal projections release oxytocin into the bloodstream. Conversely, dendritic release of the same peptide occurs within the CNS independently from axonal exocytosis (Ludwig and Leng, 2006). Clearly, polarized trafficking in these neurons has functional importance.

The consequence of disrupting the trafficking patterns of dense core granules is exemplified by the Val66Met mutation in the BDNF prohormone. This polymorphism prevents dendritic targeting of BDNF and is associated with reduced episodic memory (Egan et al., 2003). BDNF is found in presynaptic and postsynaptic compartments (Conner et al., 1997; An et al., 2008). The signals that regulate the distribution of the BDNF-containing granules are complex; dendritic localization is determined by the $3^{\prime}$-UTR and an exon-located targeting signal (An et al., 2008; Chiaruttini et al., 2009). Thus, the trafficking of dense core granules is surprisingly variable in comparison to the synaptic vesicles that contain classical transmitters. In addition to the transmitterspecific trafficking signals, might the characteristics of granular trafficking also be determined by the neuronal cell type?

The most direct way to address this question would be to compare the trafficking of the same neuropeptide in two different types of neurons. We have therefore examined the distribution of neuropeptide Y-containing dense core granules in hippocampal and hypothalamic GABAergic interneurons. This peptide was chosen because it is a prototypical peptide that is synthesized endogenously in both of these regions. Using this approach, we conclude that the characteristics of peptide trafficking can also vary between different neuronal populations. 


\section{Materials and Methods}

Cell culture. Hippocampal (Mitchell et al., 2008) and hypothalamic neurons from C57BL/6J mice [postnatal days (P) 1-3] of either sex were maintained in inverted cultures over purified cortical astrocytes $(\mathrm{P} 1-3$, DIV $>7)$ in Neurobasal/B27 medium with $0.5 \mathrm{~mm}$ L-glutamine. Cultures were used 8-14 d after plating. INS-1 cells were cultured and transfected as described previously (Whim, 2011).

Neuronal transfection and reverse transcription $P C R$. Neurons were transfected 5-8 d after plating with $1.5 \mu \mathrm{g}$ of pNPY-Venus using calcium phosphate (Mitchell et al., 2008) and fixed 2-4 d later. The agouti-related protein (AgRP) expression plasmid was made by subcloning the mouse AgRP cDNA (clone identifier 30611244; Open Biosystems) into pIRES2-AcGFP1 (Clontech). Reverse transcription (RT)-PCR was as described previously (Ramamoorthy and Whim, 2008) and used $200 \mathrm{ng}$ of mRNA from hippocampal and hypothalamic tissue (P22). AgRP primers were previously described (van den Pol et al., 2009). For the PCR, a ratio of 1:10 (hypothalamic:hippocampal) cDNA was used.

In vitro immunocytochemistry. Cultures were processed as described previously (Ramamoorthy and Whim, 2008). For double-staining experiments, antibodies were applied sequentially. Control experiments showed no significant bleed-through of the fluorescent labels or crossreactivity between antibodies. Images were obtained with a Nikon TE2000U microscope with a $60 \times$ oil-immersion objective and a Retiga 1300 monochrome camera.

In situ immunohistochemistry. Coronal brain sections were prepared from $\mathrm{P} 8-13$ mice [wildtype C57BL/6J or NPY(GFP) transgenic animals; van den Pol et al., 2009)] as detailed previously (Ramamoorthy and Whim, 2008). For double staining experiments, primary antibodies were coapplied followed by coapplication of the secondary antibodies. Images were taken with an Olympus Fluoview 300 (Pennsylvania State University, Huck Institutes, State College, PA) or a Leica DM IRE2 (Louisiana State University Health Science Center, New Orleans, LA) confocal microscope.

Sources of antibodies. Primary antibodies were rabbit anti-NPY (1:200; Peninsula Laboratories), sheep anti-NPY (1:200; Millipore), rabbit antisynapsin (1:200; Millipore), guinea pig anti-VGAT (1:1000; Millipore), goat anti-AgRP (1:200; Santa Cruz Biotechnology), and mouse anti-MAP2 (1: 200; Millipore). Secondary antibodies were donkey anti-rabbit TRITC, donkey anti-mouse TRITC, goat anti-mouse FITC, donkey anti-guinea pig FITC, donkey anti-sheep FITC, and donkey anti-goat TRITC (1:100; Jackson ImmunoResearch). The specificity of the rabbit NPY antibody was confirmed by first testing that staining was absent in NPY knock-out mice and then showing that transfection of an NPY-containing plasmid conferred NPY-immunoreactivity (ir) onto cerebellar neurons that normally lack expression (data not shown).

Image analysis. Images were analyzed using ImagePro Plus (Media Cybernetics), OriginPro7 (MicroCal Software), and Excel as described previously (Ramamoorthy and Whim, 2008).

\section{Results}

NPY-ir puncta have a differential distribution in hippocampal versus hypothalamic neurons in vitro

Using a selective antibody (see Materials and Methods), we examined the distribution of NPY in hippocampal and hypothabars, $20 \mu \mathrm{m}$

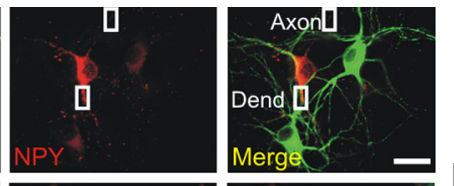

B

NPY-ir in hippocampal neurons

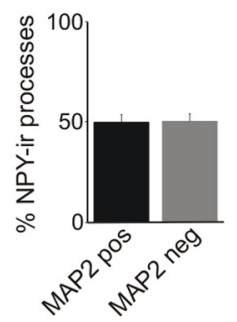

E.
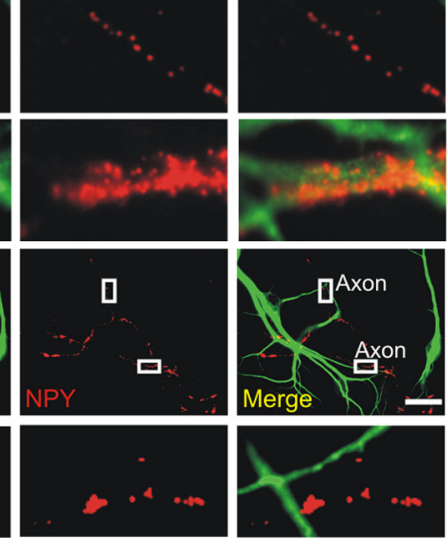

$\mathrm{D}$

NPY-ir in hypothalamic neurons
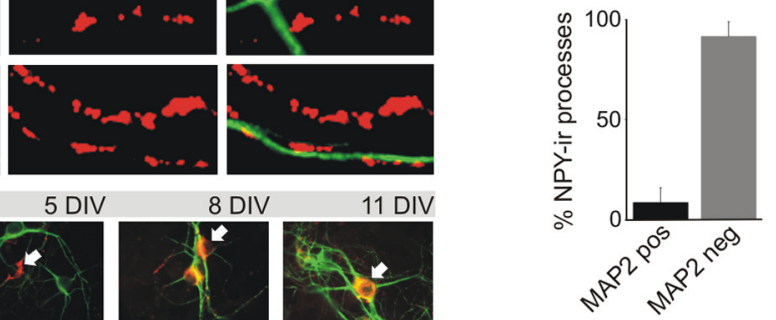

5 DIV 8 DIV

11 DIV
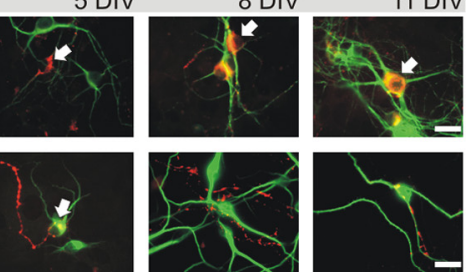

Figure 1. NPY-ir puncta have a differential distribution in hippocampal (Hippo) versus hypothalamic neurons (Hypo) in vitro. $\boldsymbol{A}_{t}$ Hippocampal neurons costained for NPY and MAP2. Boxed regions show that NPY-ir puncta are found in the axon (MAP2-negative

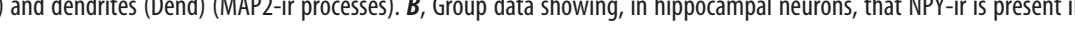
(me MAP2-negative processes (mean $\pm S D ; 67$ measurements from $n=3$ cultures). $E, F$, Somatic NPY-ir (arrows) is present in hippocampal neurons at all time points examined, but in hypothalamic (MAP2-negative) processes by 8 DIV. Since peptide synthesis occurs in the soma, the absence of somatic staining suggests that newly synthesized NPY is rapidly transported to the axon in hypothalamic neurons. Scale

lamic neurons at 8-11 DIV. These regions endogenously express NPY (Chronwall et al., 1985; Kohler et al., 1986). The cells were costained for MAP2, a protein found in the soma and dendrites of CNS neurons (Dotti et al., 1988). In hippocampal neurons, NPY-ir was found in the cell body as well as MAP2-positive and negative processes, consistent with a distribution in both axons and dendrites (Fig. 1 $A, B$ ). In contrast, in hypothalamic neurons, NPY-ir was observed in axonal (MAP2 negative) processes but rarely in the cell body or dendrites (Fig. $1 C, D$ ). When NPY-ir was examined at earlier time points in culture, immunoreactivity was prominent in the somata of hippocampal neurons from 2 to 11 DIV (Fig. 1E). However, while somatic staining was present in hypothalamic neurons up to 5 DIV, NPY-ir was restricted to MAP2-negative processes by 8-11 DIV (Fig. $1 F$ ). Thus, in polarized hippocampal neurons NPY is found in axons and dendrites while in hypothalamic neurons NPY-ir is mainly present in axons.

\section{Differential trafficking of NPY-ir puncta is also seen in CNS neurons in situ}

To determine whether the differential distribution in vitro reflected a genuine difference in neuropeptide trafficking, brain 

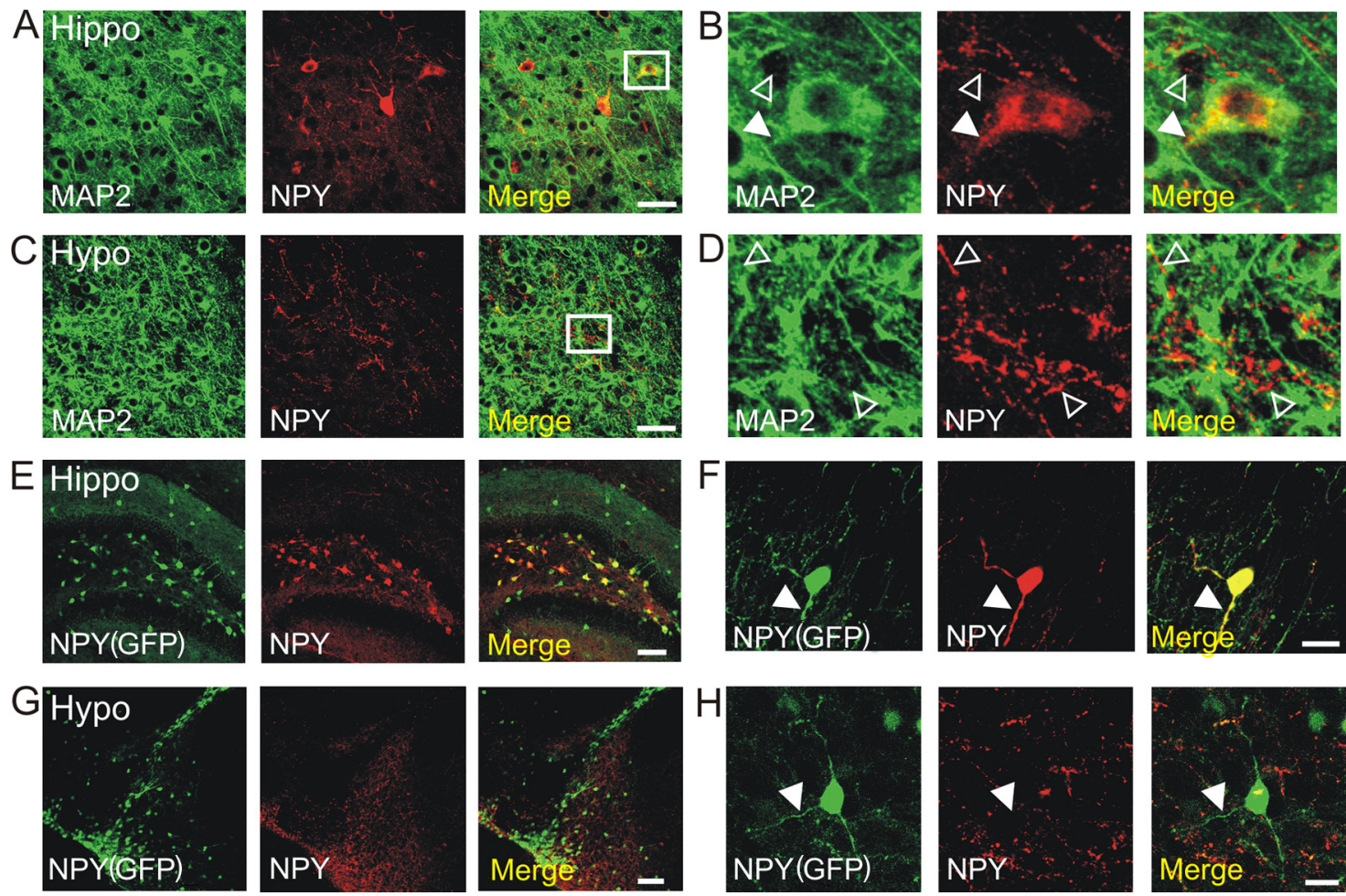

Figure 2. Differential trafficking of NPY-ir puncta in CNS neurons in situ. $\boldsymbol{A}$, Coronal section of hippocampus (Hippo) costained for MAP2 and NPY. $\boldsymbol{B}$, Magnification of boxed region in $\boldsymbol{A}$ shows NPY-ir in a neuronal cell body and a MAP2-ir dendrite (filled triangles). In the same field of view, NPY-ir puncta are also seen in an axon/MAP2-negative process (open triangles). $C$, Hypothalamic region (Hypo) (from the same slice as $\boldsymbol{A}$ ) costained for MAP2 and NPY. D, Magnification of the boxed region in $\boldsymbol{C}$ illustrates that NPY-ir processes (open triangles) do not overlap with MAP2-ir, consistent with the presence of NPY in axons, not dendrites, in hypothalamic neurons. $\boldsymbol{E}$, Coronal section of hippocampus from an NPY(GFP) transgenic mouse. $\boldsymbol{F}$, NPY-ir is present in the cell body and two processes of a GFP-expressing hippocampal neuron (filled triangle indicates one process). $\boldsymbol{G}$, Coronal section showing the hypothalamic arcuate nucleus in a NPY(GFP) transgenic mouse. $\boldsymbol{H}$, NPY-ir processes do not colocalize with GFP, which is present in the cell body and proximal processes (filled triangle) of an arcuate hypothalamic neuron. Scale bars: $\boldsymbol{A}, \boldsymbol{C}, 10 \mu \mathrm{m} ; \boldsymbol{E}, \boldsymbol{G}, 100 \mu \mathrm{m} ; \boldsymbol{F}$, H, $20 \mu \mathrm{m}$.

sections were costained for NPY and MAP2. The results confirmed the observations made in vitro. In the hippocampus, NPY-ir was observed in MAP2-positive and -negative processes and in the cell body (Fig. $2 A, B$ ). In contrast, in the hypothalamus NPY-ir was not present in neuronal cell bodies, and no convincing colocalization with MAP2-ir was seen. Rather, the NPY-ir processes were fine-beaded structures consistent with the presence of NPY in axons (Fig. 2C,D).

Because of the density of the MAP2-ir, it was difficult to trace NPY-ir within the multiple processes of a single hippocampal neuron. Therefore, we also examined NPY-ir using a transgenic mouse in which NPY neurons express cytoplasmic GFP (van den Pol et al., 2009). Figure $2 E$ shows a low-power view of GFP and NPY-ir in the hippocampus. In the cell shown in Figure $2 F$, NPY-ir can be observed in two GFP-containing processes. Thus, we can minimally conclude that NPY-containing granules are present in hippocampal dendrites (at least one of the processes must be a dendrite). Similar experiments in the hypothalamic arcuate nucleus revealed that the fine NPY-ir did not convincingly colocalize with GFP-labeled cells bodies or processes (Fig. $2 G, H)$. A possible interpretation is that GFP is not present in distal axonal processes. Nevertheless, these results are consistent with the idea that NPY is differentially localized in hippocampal and hypothalamic neurons.

NPY-ir differentially colocalizes with axonal markers in hippocampal and hypothalamic neurons

To confirm the expression of NPY in hippocampal and hypothalamic axons, cultured neurons were costained for NPY and the vesicular GABA transporter (VGAT). The latter is a marker of presynaptic GABAergic varicosities (Chaudhry et al., 1998). The majority of NPY-ir processes from both the hippocampus and the hypothalamus were VGAT-ir ( 81 and $86 \%$, respectively; $>63$ processes were analyzed from one culture). VGAT-ir puncta colocalized with NPY-ir puncta in both types of neurons (Fig. $3 A, B)$. Quantification indicated that the majority of axonal NPY-ir puncta colocalized with VGAT-ir in hippocampal (94 $\pm 10 \%$, mean $\pm \mathrm{SD} ; 10$ processes from $n=3$ cultures $)$ and hypothalamic neurons $(99 \pm 1 \%$, mean $\pm \mathrm{SD} ; 10$ processes from $n=3$ cultures). Thus, most of the presynaptic NPY puncta were located at synaptic varicosities.

These results are consistent with an axonal and dendritic distribution of NPY in hippocampal neurons. Conversely, in hypothalamic neurons NPY was present in the axon with negligible dendritic expression. To more precisely define the location of the NPY-ir puncta, cultures were costained for synapsin, a presynaptic vesicle protein. In the axons of hippocampal neurons most NPY-ir puncta colocalized with synapsin-ir puncta (Fig. 3C), indicating that NPY-containing granules were clustered at the sites of classical transmitter release. The low levels of NPY-ir in the interbouton regions may reflect a low level of spontaneous NPY secretion in culture (and hence little need for store replenishment). In the dendrites, about half of the NPY puncta were located opposite synapsin puncta. This suggests that NPY might be released dendritically in a location that could regulate presynaptic transmitter release (Fig. 3C, bottom). In contrast, in hypothalamic neurons almost all NPY-ir puncta were colocalized with 
synapsin-ir (Fig. 3D). These results are again consistent with the pattern of NPY distribution seen in the brain slices.

\section{Heterologously expressed NPY mimics the polarized trafficking of endogenous NPY}

The cell type-specific trafficking of NPY implies that the prohormone does not play a major role in determining granular distribution. To test this idea, we took two approaches. First, we examined the distribution of NPY-containing puncta in cells transfected with an NPY prohormone tagged with Venus. In transfected hippocampal neurons, the NPY-Venus puncta were present in the cell body and axonal and dendritic compartments (Fig. 4A, C). In hypothalamic neurons, NPY-Venus puncta were found almost exclusively in the axon/ MAP2-negative compartment (Fig. 4B,C). Thus, the heterologously expressed NPY shows the same distribution as endogenous neuropeptide $\mathrm{Y}$, even though the fusion construct contains only the minimal preprohormone required for peptide expression (i.e., lacks the NPY promoter and an intact $3^{\prime}$-UTR).

Agouti-related protein shows the same differential trafficking pattern as NPY Second we examined whether the subcellular distribution of another polypeptide matched that of NPY. For these experiments we looked at the expression of $\mathrm{AgRP}$, since this polypeptide is present in the hypothalamic arcuate neurons that synthesize NPY (Broberger et al., 1998).

When AgRP-ir was examined in hippocampal cultures, it was apparent that a subset of the NPY-ir neurons were also AgRP-ir (34 $\pm 13 \%$, mean \pm SD; 60 neurons from $n=3$ cultures). Some cells were costained (Fig. 4D) while others lacked AgRP-ir (Fig. 4E). However, all of the AgRP-ir neurons were NPY-ir. In these neurons AgRP-ir was colocalized with NPY-ir throughout the cell (Fig. 4D). Since NPY is found in both axons and dendrites in hippocampal neurons (Fig. 1), this means that AgRP is also found in both of these compartments.

In hypothalamic cultures most of the NPY-ir neurons were AgRP-ir (94 $\pm 3 \%$, mean \pm SD; 67 neurons from $n=3$ cultures). In these neurons, AgRP-ir was not found in the cell body but was only observed in the NPY-ir axons (Fig. $4 F$ ).

Because hippocampal expression of AgRP was not observed previously (Broberger et al., 1998), we used RT-PCR to confirm that AgRP mRNA was present in hippocampal tissue, albeit at lower levels than in the hypothalamus (Fig. 4G). In addition, the AgRP antibody was selective, because when the INS-1 endocrine cell line was transfected with a plasmid encoding mouse AgRP, punctate immunoreactivity was only observed in transfected cells (Fig. $4 H$ ) and no staining was seen when the primary antibody was omitted (Fig. 4I). Finally, although INS-1 cells express NPY
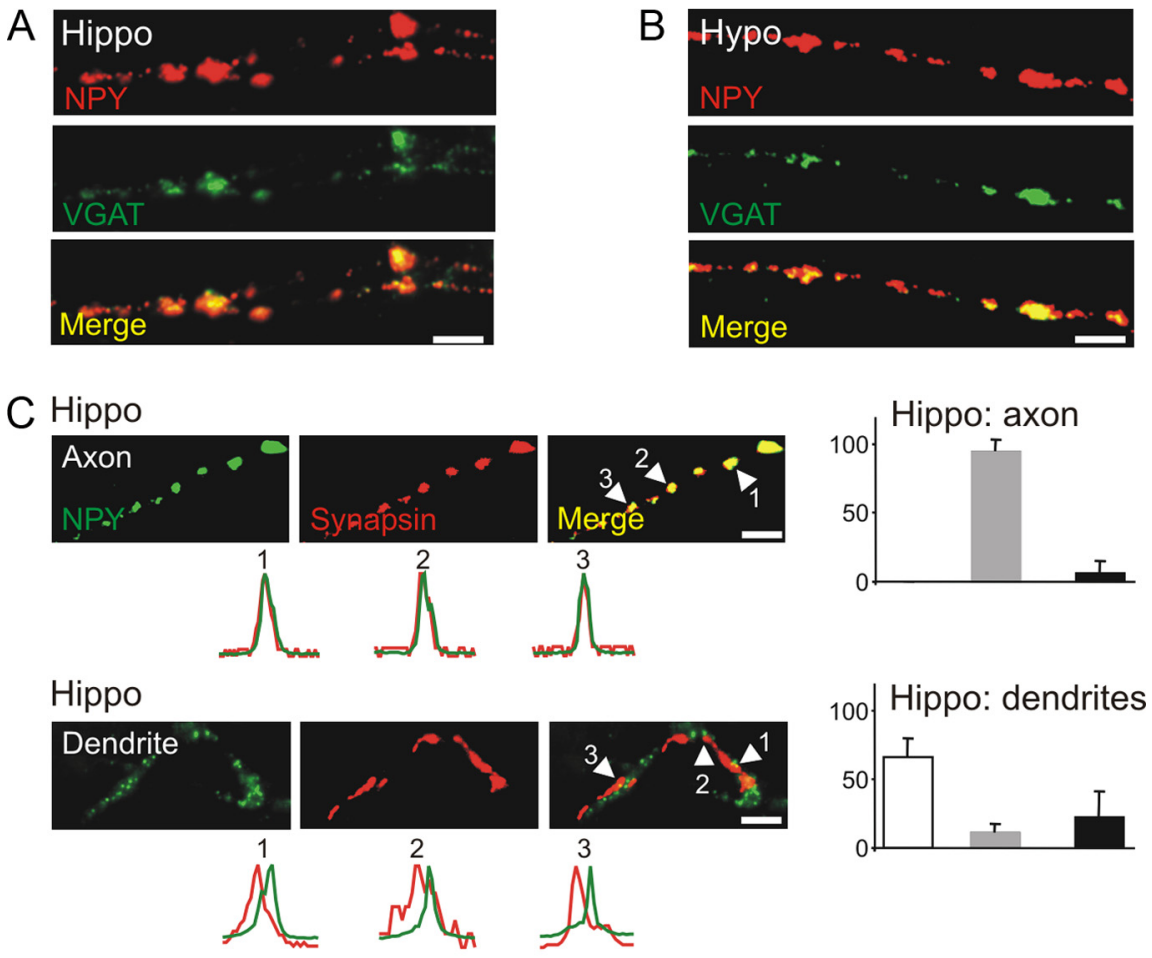

Figure 3. NPY-ir differentially colocalizes with axonal and synaptic markers in hippocampal (Hippo) and hypothalamic neurons (Hypo) in vitro. A, NPY-ir puncta are colocalized with axonal VGAT-ir varicosities in hippocampal neurons. $\boldsymbol{B}$, In hypothalamic 作 axon. Bottom panel is an example of an NPY-ir process that is juxtaposed to synapsin-ir varicosities. This is consistent latter are present in axons. Group data are shown in the right column (mean \pm SD; $1-8$ neurons quantified from $n=3$ cultures). Line scans were normalized to the maximum signal in each fluorescent channel. Scale bars, $4 \mu \mathrm{m}$.

(Whim, 2011), none of the NPY-ir cells were AgRP-ir, confirming that the antibodies did not cross-react (Fig. $4 \mathrm{~J}$ ).

These results are therefore consistent with the idea that NPY trafficking can be determined on a cell-type basis (rather than solely by the characteristics of the peptide per se).

\section{Discussion}

Here we have investigated the subcellular distribution of NPY-ir within hippocampal and hypothalamic neurons. Our primary conclusion is that NPY trafficking is cell type dependent. In hippocampal neurons NPY-ir puncta are present in the axon and dendrites. In contrast, in hypothalamic neurons NPY-ir puncta are present almost exclusively in the axon. Four pieces of evidence suggest that this arises from a fundamental property of certain classes of neurons or perhaps granules (see below). First, differential trafficking is observed in neurons in situ under minimally disturbed conditions. Second, the same features are observed when the neurons are maintained in cell culture. Third, the distribution of heterologously expressed NPY mimics that of endog- 

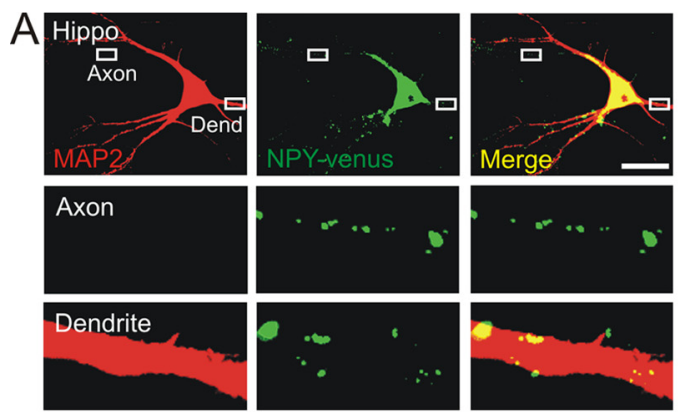

C

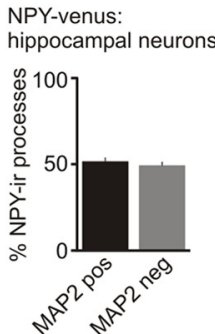

NPY-venus:

hypothalamic neurons

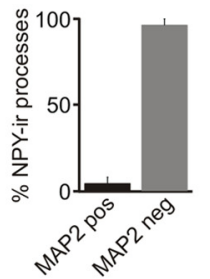

G

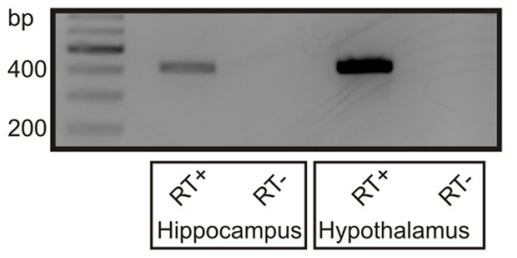

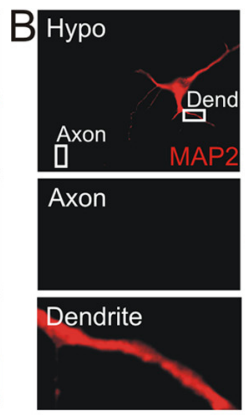
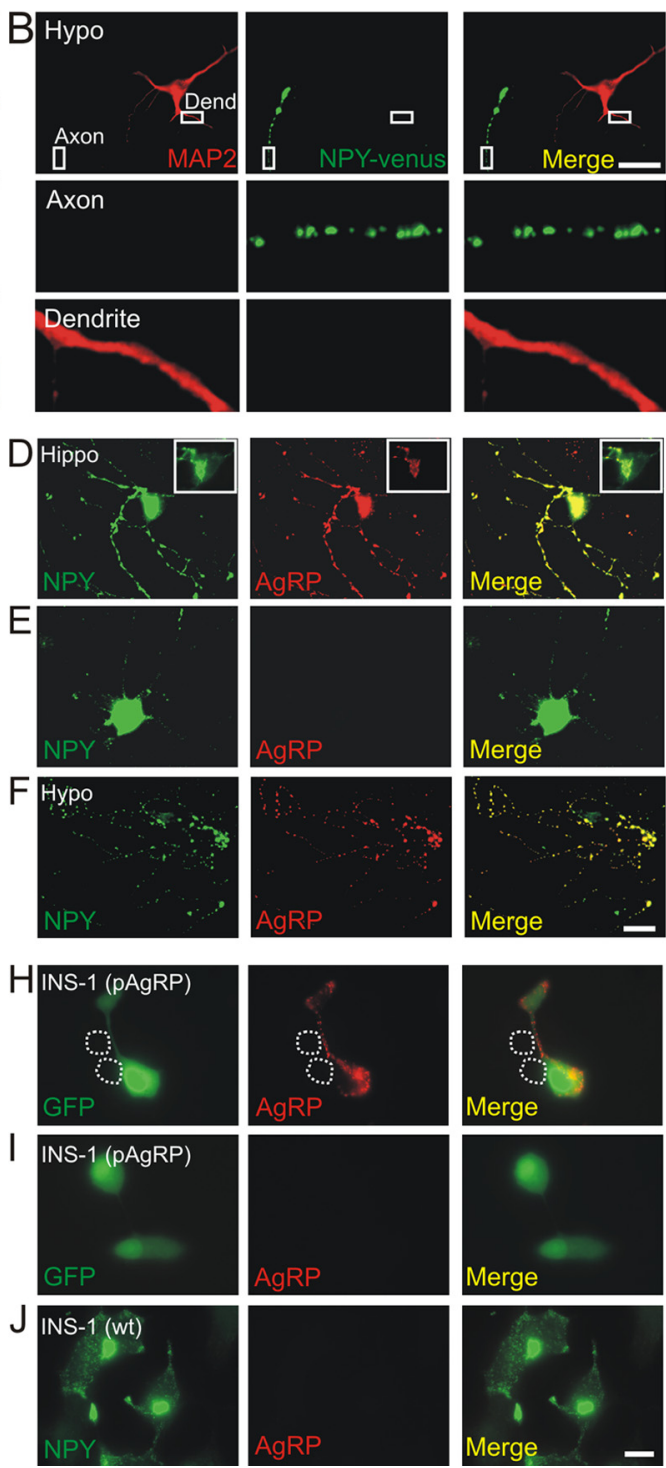

Figure 4. The NPY prohormone does not play a major role in determining granular distribution. Heterologously expressed neuropeptide $Y(\boldsymbol{A}-\boldsymbol{C})$ and endogenous AgRP $(\boldsymbol{D}-\boldsymbol{F})$ show the same cell type-specific trafficking as endogenous NPY.A, A transfected hippocampal neuron (Hippo) expressing NPY-Venus. Costaining with MAP2 reveals that the NPY-Venus puncta are present in the MAP2-ir dendrites and in the MAP2-negative axon. $\boldsymbol{B}$, Example of an NPY-Venus-expressing process from a transfected hypothalamic neuron (Hypo). Note that no NPY-Venus-labeled puncta are present in the soma or dendrites (MAP2-ir processes). However, NPY-Venus puncta are visible in the axonal process that projects from the MAP2-ir cell. C, Group data for heterologous expression of NPY-Venus (mean \pm SD; $10-22$ neurons quantified from $n=3$ cultures). $D$, In hippocampal cultures a subset of NPY-ir neurons was also AgRP-ir. In these neurons the NPY-it and AgRP-ir were colocalized. Thus, in hippocampal neurons AgRP is present in axons and dendrites. Insets show somatic staining. $\boldsymbol{E}$, Some NPY-ir hippocampal neurons lacked AgRP-ir. $\boldsymbol{F}$, In hypothalamic cultures NPY-ir processes are also AgRP-ir. Since NPY in hypothalamic neurons is present in the axon, AgRP-ir must also be restricted to this compartment. $\mathbf{G}$, RT-PCR experiment showing that AgRP mRNA is present in the hippocampus and hypothalamus. $\boldsymbol{H}$, INS-1 cells transfected with pAgRP-IRES-GFP are AgRP-ir, whereas no staining is seen in two GFP-negative cells (white circles). I, No AgRP-ir was observed in transfected cells when the primary AgRP antibody was omitted.J, Untransfected INS-1 cells are NPY-ir but not AgRP-ir, confirming that the antibodies do not cross-react. Scale bars: $A, B, 30 \mu \mathrm{m} ; \boldsymbol{D}, \boldsymbol{E}, 20 \mu \mathrm{m} ; \boldsymbol{H}-\boldsymbol{J}, 10 \mu \mathrm{m}$.

enous NPY. Fourth, granular distribution is not determined by the neuropeptide prohormone, because both neuropeptide $\mathrm{Y}$ and AgRP-containing granules show the same distribution in different neurons. These features argue that the difference in granular trafficking between hippocampal and hypothalamic neurons is not an experimentally induced artifact (point one). It is an intrinsic property of these neurons (points 2 and 3), and cell-type differential trafficking is not limited to a single polypeptide (point 4).

How do these results compare to those of other neuropeptides from the same brain regions? The hypothalamic peptides oxytocin and vasopressin are transported to axonal terminals and released as hormones into the blood. They are also found in dendrites and released within the CNS (Ludwig and Leng, 2006). This distribution appears very different from that of NPY. The hypothalamic neurons that we studied in situ and in vitro sent the NPY-containing granules only to the axon. Curiously, however, an "oxytocin-vasopressinlike" distribution was found when NPY was examined in hippocampal neurons. In this brain region NPY-containing granules were present in the axons and dendrites, consistent with an earlier study (Deller and Leranth, 1990). A dendritic and axonal localization has been shown for BDNF (Hartmann et al., 2001) in hippocampal neurons. However, only a small fraction of BDNF granules cluster at presynaptic release sites (Swanwick et al., 2004) in contrast to most NPY-containing granules, perhaps reflecting a difference in function (neurotrophin vs classical neuromodulator). Many neuropeptides, including CGRP and somatostatin, are present in hippocampal axons (Kunkel et al., 1994; Freund et al., 1997), but whether they are also present in dendrites is less clear. 
While the dendritic localization of peptide appears to be quite rare, neuropeptides are often localized after colchicine injection to prevent microtubule-based transport. This increases somatic immunoreactivity but is also likely to alter the relative distribution of peptidergic granules. Dendritic localization of peptides has been reported in the hypothalamus and locus coeruleus (Oldfield et al., 1985; Vila-Porcile et al., 2009). Dendritic trafficking may therefore be more common than is generally appreciated.

The colocalization of NPY-ir and synapsin-ir in hippocampal and hypothalamic neurons suggests that presynaptic peptide release occurs from the same varicosities that release classical transmitters. Most of the hippocampal NPY-synthesizing cells we studied were GABAergic. In these cells, NPY has been found in axonal and dendritic processes (Deller and Leranth, 1990). Exogenous NPY application presynaptically inhibits hippocampal glutamatergic transmission (Qian et al., 1997). Release of axonal NPY could play this modulatory role.

The function of the dendritic NPY that we observed is not clear. However some mossy fibers make synaptic connections onto dendrites of NPY-ir neurons (Deller and Leranth, 1990). The dendritic NPY-ir puncta were often in close proximity to synapsin-ir varicosities, suggesting that dendritic release of NPY could modulate presynaptic glutamate release. The postsynaptic release of BDNF from hippocampal neurons has been demonstrated (Hartmann et al., 2001).

In the hypothalamus, NPY-synthesizing neurons are mainly located within the arcuate nucleus (Chronwall et al., 1985), and such neurons regulate food intake (Luquet et al., 2005). The axonal location of hypothalamic NPY that we observed is consistent with a study showing that axonal varicosities of neurons in the arcuate nucleus contain GABAergic synaptic vesicles and NPYcontaining granules (Cowley et al., 2001).

The mechanism underlying the cell type-dependent trafficking that we observed is not known. Presumably it reflects the existence of distinct populations of granules, only some of which can access the dendritic compartment. It is clear from work on other preparations that subsets of dense core granules do exist (Landry et al., 2003).

Although the mechanisms that guide neuropeptides into dense core granules are the subject of debate, the diversity in targeting we observed occurs with a single polypeptide. Thus, cell-type-specific factors probably contribute to directing the type of trafficking that we describe here.

\section{References}

An JJ, Gharami K, Liao GY, Woo NH, Lau AG, Vanevski F, Torre ER, Jones KR, Feng Y, Lu B, Xu B (2008) Distinct role of long 3' UTR BDNF mRNA in spine morphology and synaptic plasticity in hippocampal neurons. Cell 134:175-187.

Blomqvist AG, Herzog H (1997) Y-receptor subtypes-how many more? Trends Neurosci 20:294-298.

Broberger C, Johansen J, Johansson C, Schalling M, Hökfelt T (1998) The neuropeptide $\mathrm{Y} /$ agouti gene-related protein (AGRP) brain circuitry in normal, anorectic, and monosodium glutamate-treated mice. Proc Natl Acad Sci U S A 95:15043-15048.

Chaudhry FA, Reimer RJ, Bellocchio EE, Danbolt NC, Osen KK, Edwards RH, Storm-Mathisen J (1998) The vesicular GABA transporter, VGAT, localizes to synaptic vesicles in sets of glycinergic as well as GABAergic neurons. J Neurosci 18:9733-9750.

Chiaruttini C, Vicario A, Li Z, Baj G, Braiuca P, Wu Y, Lee FS, Gardossi L, Baraban JM, Tongiorgi E (2009) Dendritic trafficking of BDNF mRNA is mediated by translin and blocked by the G196A (Val66Met) mutation. Proc Natl Acad Sci U S A 106:16481-16486.

Chronwall BM, DiMaggio DA, Massari VJ, Pickel VM, Ruggiero DA,
O'Donohue TL (1985) The anatomy of neuropeptide-Y-containing neurons in rat brain. Neuroscience 15:1159-1181.

Conner JM, Lauterborn JC, Yan Q, Gall CM, Varon S (1997) Distribution of brain-derived neurotrophic factor (BDNF) protein and mRNA in the normal adult rat CNS: evidence for anterograde axonal transport. J Neurosci 17:2295-2313.

Cowley MA, Smart JL, Rubinstein M, Cerdán MG, Diano S, Horvath TL, Cone RD, Low MJ (2001) Leptin activates anorexigenic POMC neurons through a neural network in the arcuate nucleus. Nature 411:480-484.

Deller T, Leranth C (1990) Synaptic connections of neuropeptide Y (NPY) immunoreactive neurons in the hilar area of the rat hippocampus. J Comp Neurol 300:433-447.

Dotti CG, Sullivan CA, Banker GA (1988) The establishment of polarity by hippocampal neurons in culture. J Neurosci 8:1454-1468.

Egan MF, Kojima M, Callicott JH, Goldberg TE, Kolachana BS, Bertolino A, Zaitsev E, Gold B, Goldman D, Dean M, Lu B, Weinberger DR (2003) The BDNF val66met polymorphism affects activity-dependent secretion of BDNF and human memory and hippocampal function. Cell 112:257-269.

Freund TF, Hájos N, Acsády L, Görcs TJ, Katona I (1997) Mossy cells of the rat dentate gyrus are immunoreactive for calcitonin gene-related peptide (CGRP). Eur J Neurosci 9:1815-1830.

Hartmann M, Heumann R, Lessmann V (2001) Synaptic secretion of BDNF after high-frequency stimulation of glutamatergic synapses. EMBO J 20:5887-5897.

Huang LY, Neher E (1996) $\mathrm{Ca}^{2+}$-dependent exocytosis in the somata of dorsal root ganglion neurons. Neuron 17:135-145.

Köhler C, Eriksson L, Davies S, Chan-Palay V (1986) Neuropeptide Y innervation of the hippocampal region in the rat and monkey brain. J Comp Neurol 244:384-400.

Kunkel DD, Nguyen LB, Harrigan MR, Schwartzkroin PA, Malouf AT (1994) Somatostatin-containing neurons in rat organotypic hippocampal slice cultures: light and electron microscopic immunocytochemistry. Hippocampus 4:157-166.

Landry M, Vila-Porcile E, Hökfelt T, Calas A (2003) Differential routing of coexisting neuropeptides in vasopressin neurons. Eur J Neurosci 17:579-589.

Ludwig M, Leng G (2006) Dendritic peptide release and peptide-dependent behaviours. Nat Rev Neurosci 7:126-136.

Luquet S, Perez FA, Hnasko TS, Palmiter RD (2005) NPY/AgRP neurons are essential for feeding in adult mice but can be ablated in neonates. Science 310:683-685.

Mitchell GC, Wang Q, Ramamoorthy P, Whim MD (2008) A common single nucleotide polymorphism alters the synthesis and secretion of neuropeptide Y. J Neurosci 28:14428-14434.

Oldfield BJ, Hou-Yu A, Silverman AJ (1985) A combined electron microscopic HRP and immunocytochemical study of the limbic projections to rat hypothalamic nuclei containing vasopressin and oxytocin neurons. J Comp Neurol 231:221-231.

Qian J, Colmers WF, Saggau P (1997) Inhibition of synaptic transmission by neuropeptide $\mathrm{Y}$ in rat hippocampal area CA1: modulation of presynaptic $\mathrm{Ca}^{2+}$ entry. J Neurosci 17:8169-8177.

Ramamoorthy P, Whim MD (2008) Trafficking and fusion of neuropeptide Y-containing dense-core granules in astrocytes. J Neurosci 28:13815-13827.

Shakiryanova D, Tully A, Levitan ES (2006) Activity-dependent synaptic capture of transiting peptidergic vesicles. Nat Neurosci 9:896-900.

Sobota JA, Mohler WA, Cowan AE, Eipper BA, Mains RE (2010) Dynamics of peptidergic secretory granule transport are regulated by neuronal stimulation. BMC Neurosci 11:32.

Swanwick CC, Harrison MB, Kapur J (2004) Synaptic and extrasynaptic localization of brain-derived neurotrophic factor and the tyrosine kinase $\mathrm{B}$ receptor in cultured hippocampal neurons. J Comp Neurol 478:405-417.

van den Pol AN, Yao Y, Fu LY, Foo K, Huang H, Coppari R, Lowell BB, Broberger C (2009) Neuromedin B and gastrin-releasing peptide excite arcuate nucleus neuropeptide $\mathrm{Y}$ neurons in a novel transgenic mouse expressing strong Renilla green fluorescent protein in NPY neurons. J Neurosci 29:4622-4639.

Vila-Porcile E, Xu ZQ, Mailly P, Nagy F, Calas A, Hökfelt T, Landry M (2009) Dendritic synthesis and release of the neuropeptide galanin: morphological evidence from studies on rat locus coeruleus neurons. J Comp Neurol 516:199-212.

Whim MD (2011) Pancreatic beta cells synthesize neuropeptide $Y$ and can rapidly release peptide cotransmitters. PLoS One 6:e19478. 